\title{
Late collapse of distal radius fractures after K-wire removal: is it significant?
}

\author{
H. V. Kurup · V. M. Mandalia · K. A. Shaju • \\ B. Singh $\cdot$ A. R. Beaumont
}

Received: 12 November 2007 / Accepted: 1 April 2008/Published online: 22 May 2008

(C) Springer-Verlag 2008

\begin{abstract}
Background The aim of this study was to find out whether distal radius fractures treated by Kirschner wire $(\mathrm{K}$ wire) fixation loose reduction after wire removal and analyze the variables may influence this.

Materials and methods Patients who underwent $\mathrm{K}$ wire fixation for unstable fractures of distal radius over a period of 3 years were included in this retrospective study. Fractures were classified according to AO classification. Radiographs taken just prior to removal of $\mathrm{K}$ wires and radiographs taken at least 1 month after wire removal were analyzed to study three radiological parameters; Palmar or dorsal tilt, radial inclination and ulnar variance. Loss of these angles was analyzed statistically against variables like age, sex, AO classification and duration of fixation.

Results 59 fractures were analyzed with mean age of 56 years and male to female ratio of 1:2. Average loss of radial tilt was $2.6^{\circ}$, loss of palmar tilt was $2.6^{\circ}$ and loss of ulnar variance was $1.3 \mathrm{~mm}$.

Conclusions We found that distal radius fractures treated by percutaneous $\mathrm{K}$ wire fixation, did not suffer significant loss of reduction of fracture position after removal of wires. This remains true regardless of age, sex, fracture type according to AO type or duration of wire fixation.
\end{abstract}

H. V. Kurup ( $\square)$

Mayday University Hospital, Croydon CR7 7YE, UK

e-mail: harishvk@yahoo.com

V. M. Mandalia · K. A. Shaju - A. R. Beaumont

Salisbury District Hospital, Salisbury SP2 8BJ, UK

B. Singh

Queen Elizabeth Hospital, Woolwich, London SE18 4QH, UK
Keywords Distal Radius Fracture $\cdot \mathrm{K}$ wire fixation · Loss of reduction - Radiological variables

\section{Introduction}

Fractures of the distal radius are the most common of all orthopedic injuries accounting for nearly $20 \%$ of all fractures presenting to emergency department [1]. Malunion of distal radius fractures lead to posttraumatic arthritis, mid carpal instability and pain [2]. Conservative treatment of minimally displaced and stable fractures of distal end of radius in elderly patients usually shows a good outcome but the treatment of severely displaced and unstable fractures has been controversial. The functional results in patients with significant radial shortening are poor. Fujii et al. [3] recommended reduction of these fractures even in elderly patients. Percutaneous pinning is said to be a simple, minimally invasive technique and is aimed at preventing redisplacement of the fragments. Wires are usually retained for 4-6 weeks. Most opponents of this technique claim that these fractures tend to collapse even after removal of wires [4]. The aim of our study was to find out whether these fractures loose reduction after removal of wires and whether this has any statistically significant association with any of the factors looked at.

\section{Materials and methods}

Patients who underwent Kirschner wire (K wire) fixation for distal radius fractures in our department from June 2000 to May 2003 were included in this retrospective study. Palmar Tilt, Radial Tilt and Ulnar Variance were measured in these patients on plane radiographs [5]. Extra-articular 
fractures of Colles type and intra-articular fractures except Barton type were included in this study. The exclusion criteria were pediatric fractures, additional procedures like external fixation and non-availability of satisfactory and comparable radiographs for measurement. Both bicortical $\mathrm{K}$ wire fixations with radial or dorsal entry points and Kapandji wire fixations were included in this study. All operations were carried out by consultants or registrars with appropriate experience. Fractures were fixed within 121 days (mean 4.78 days). $\mathrm{K}$ wires were removed in outpatient clinic usually between 4 and 6 weeks depending on the preference of the consultant surgeon in charge of the patient. The fractures were classified using AO classification. The loss of palmar tilt, radial tilt and ulnar variance were measured from radiographs done prior to wire removal and later in follow up (3-4 months after injury and at least 1 month after wire removal). Only patients who were reviewed at a later date in clinic with a radiograph were included in this study. Out of a total 113 patients, 54 had to be excluded based on these criteria.

The variables studied were age, sex, fracture type (AO), presence of ulnar styloid fracture and period of wire stabilization. Statistical analysis was done using SPSS 10.0 software (SPSS Inc. Chicago, IL, USA). $P$ value $<0.05$ was considered to be significant for the purpose of this study.

\section{Results}

Fifty-nine patients were included in this study of which 39 (66\%) were females and 20(34\%) males. Mean age of patients was 56 years (range 18-86 years with a median age of 59 years). We divided patients into two age groups ( $<65$ years and $>$ or $=65$ years). $56 \%$ were $<65$ years old and $44 \%$ were $\geq 65$. Age and sex (Table 1) had no influence on the loss of radiological parameters after wire removal.

Fractures were classified using $\mathrm{AO}$ system. No patients were in class $\mathrm{B}$, due to different treatment protocol followed in these. AO Class of the fracture had no influence on loss of angles after wire removal (Table 2). Wires were mostly removed in outpatients after 5 or 6 weeks with some at 4 and some after 6 weeks. The duration of fixation had no association with the radiological outcome (Table 3 ).

\section{Discussion}

Abraham Colles (1814) [6] first described Colles fracture and stated "one consolation only remains that the limb will at some remote period again enjoy perfect freedom in all its motions and be completely exempt from pain; the deformity however will remain undiminished through life." The different surgical techniques for treating fractures of distal radius are aimed at reducing this final deformity to ensure better anatomical results especially in more severe fractures. Most distal radial fractures are uncomplicated and effectively treated with closed reduction and immobilization in a cast. The goal of treatment is to restore the functional anatomy by a method that does not compromise hand function. The important factors to consider are fracture pattern, displacement, stability of fracture, age and physical demands of the patient [1].

Various modalities of treatment for distal radius fracture are described in the literature and the four methods generally followed in treating these fractures are, one conservative treatment in plaster cast with or without manipulative reduction; two, percutaneous $\mathrm{K}$ wire fixation to maintain reduction while in plaster; three, External fixation (bridging or non-bridging); four, open reduction and internal fixation. There is no general consensus regarding the indications, advantages and disadvantages of each of these treatment methods [7]. Percutaneous pin fixation is an excellent technique provided distal aspect of radius is not severely comminuted or osteoporotic. It is especially useful for unstable fractures both extra articular and intra articular sometimes in combination with other modes of fixation [1]. This technique was first reported by Stein and Katz [8] and further refined by Clancey [9]. K wires are placed through radial styloid and/or dorsally to aid in reduction. Alternatively they may be placed intrafocally as in Kapandji's technique [10]. Cross pinning with two radial styloid pins
Table 1 Loss of angles and age/sex

\begin{tabular}{llll}
\hline Category (number of patients) & \multicolumn{4}{l}{ Loss of angles-mean (range) } \\
\cline { 2 - 4 } & Radial tilt & Ulnar variance (mm) & Dorsal tilt $^{\circ}$ \\
\hline$<65$ years (33) & $2.2(0-12)$ & $1.1(0-4)$ & $2.2(0-12)$ \\
$\geq 65$ years (26) & $2.0(0-30)$ & $1.5(0-10)$ & $3.1(0-12)$ \\
$P$ value (Mann-Whitney test) & 0.967 & 0.927 & 0.164 \\
Male (20) & $2.3(0-12)$ & $1.4(0-4)$ & $2.1(0-12)$ \\
Female (39) & $2.8(0-30)$ & $1.3(0-10)$ & $2.9(0-12)$ \\
$P$ value (Mann-Whitney test) & 0.585 & 0.927 & 0.164 \\
All patients (59) & $2.6(0-30)$ & $1.3(0-10)$ & $2.6(0-12)$ \\
\hline
\end{tabular}


Table 2 Loss of angles and AO class

Table 3 Loss of angles and timing of wire removal

\begin{tabular}{llll}
\hline AO class (number of patients) & \multicolumn{2}{l}{ Loss of angles-mean (range) } \\
\cline { 2 - 4 } & Radial tilt $^{\circ}$ & Ulnar variance (mm) & Dorsal tilt $^{\circ}$ \\
\hline A2 (10) & $5.4(0-30)$ & $1.1(0-2)$ & $2.7(0-12)$ \\
A3 (18) & $1.7(0-4)$ & $1.2(0-4)$ & $3.4(0-12)$ \\
C1 (14) & $1.8(0-6)$ & $0.9(0-2)$ & $2.5(0-8)$ \\
C2 (12) & $1.5(0-6)$ & $1.8(0-10)$ & $1.6(0-6)$ \\
C3 (5) & $4(0-12)$ & $1.2(0-4)$ & $3.0(0-4)$ \\
$P$ value (analysis of variance) & 0.106 & 0.795 & 0.528 \\
\hline
\end{tabular}

\begin{tabular}{llll}
\hline Timing of K-wire removal (number of patients) & \multicolumn{4}{l}{ Loss of angles-mean (range) } \\
\cline { 2 - 4 } & Radial tilt $^{\circ}$ & Ulnar variance (mm) & Dorsal tilt $^{\circ}$ \\
\hline 4 weeks (8) & $1.5(0-4)$ & $0.9(0-4)$ & $1.5(0-4)$ \\
5 weeks (12) & $3.6(0-12)$ & $1.0(0-2)$ & $3.2(0-12)$ \\
6 weeks (33) & $1.8(0-6)$ & $1.2(0-8)$ & $2.7(0-12)$ \\
$>6$ weeks (6) & $6.7(0-30)$ & $2.7(0-10)$ & $2.2(0-4)$ \\
$P$ value (analysis of variance) & 0.075 & 0.235 & 0.565 \\
\hline
\end{tabular}

and placement of a pin from the ulnar corner of the radius is described to be the most rigid construct biomechanically [11]. Secondary displacement after $\mathrm{K}$ wire fixation is explained by residual instability due to fracture comminution or osteoporosis. Correlation between anatomical and functional results is yet another controversial topic in treatment of these fractures. Most authors consider loss of radial length (ulnar variance) as the most crucial radiological parameter that influences the functional outcome $[5,12]$.

AO classification is the most detailed and useful system for classification of distal radial fractures with significant inter observer agreement $[13,14]$. We selected AO classification to classify fractures in our series based on these observations.

We found that distal radial fractures did not suffer significant loss of reduction after removal of wires. We have reported in a previous study [15] that during the period of wire fixation and before wire removal there is significant loss of these angles. Average loss of radial tilt reported in that study was $4.12^{\circ}$, loss of dorsal tilt was $8.07^{\circ}$ and loss of ulnar variance was $3.12 \mathrm{~mm}$. Compared to this, the loss of angles after wire removal is significantly less. (Radial tilt: $2.6^{\circ}$; dorsal tilt : 2.6 and ulnar variance: $1.3 \mathrm{~mm}$ ). Age, sex and AO class did not influence loss of reduction after wire removal in contrast to the observation during wire fixation where they had showed statistically significant loss of radiological parameters with increasing age, female sex and increasing comminution as in AO class. Based on our observations we found that leaving wires in situ for longer than 4 weeks did not offer any significant advantage in terms of securing fracture reduction and the same was true with the use of Kapandji wires. Previous studies have shown that Kapandji wires do not offer any protection against late collapse after wire removal [4].

The limitations of our study were that being a retrospective study, we had to exclude patients without comparable radiographs for measurements and that we did not investigate into the functional outcome in these patients.

Kirschner wire fixation for unstable fractures of distal radius is a good technique to prevent redisplacement. Removal of wires can be done at any convenient point after the fourth week as practiced widely but preferably before 6 weeks. Loss of reduction after removal of wires is insignificant and is not influenced by age, fracture comminution and period of fixation.

Author's declaration This study was conducted according to the current laws in United Kingdom and in accordance with the ethical standards of the 1964 Declaration of Helsinki. Informed consent was obtained from patients where necessary.

Conflict of interest statement The authors declare that they have no conflict of interest related to the publication of this manuscript.

\section{References}

1. Simic PM, Weiland AJ (2003) Fractures of the distal aspect of the radius: changes in treatment over the past two decades. Instr Course Lect 52:185-195

2. Miyake T, Hashizume H, Inoue H, Shi Q, Nagayama N (1994) Malunited colles' fracture. Analysis of stress distribution. J Hand Surg [Br] 19(6):737-742

3. Fujii K, Henmi T, Kanematsu Y, Mishiro T, Sakai T, Terai $T$ (2002) Fractures of the distal end of radius in elderly patients: 
a comparative study of anatomical and functional results. J Orthop Surg (Hong Kong) 10(1):9-15

4. Brady O, Rice J, Nicholson P, Kelly E, O'Rourke SK (1999) The unstable distal radial fracture one year post Kapandji intrafocal pinning. Injury 30(4):251-255

5. Hollevoet N, Verdonk R (2003) The functional importance of malunion in distal radius fractures. Acta Orthop Belg 69(3): 239-245

6. Abraham Colles (1972) The classic. On the fracture of the carpal extremity of the radius. Edinburgh Med Surg J Clin Orthop 83:35

7. Handoll HH, Madhok R (2003) Surgical interventions for treating distal radial fractures in adults. Cochrane Database Syst Rev CD003209

8. Stein AH Jr, Katz SF (1975) Stabilization of comminuted fractures of the distal inch of the radius: percutaneous pinning. Clin Orthop 108:174-181

9. Clancey GJ (1984) Percutaneous Kirschner-wire fixation of Colles fractures. A prospective study of thirty cases. J Bone Joint Surg Am 66(7):1008-1014
10. Kapandji A (1987) Intra-focal pinning of fractures of the distal end of the radius 10 years later. Ann Chir Main 6(1):57-63

11. Naidu SH, Capo JT, Moulton M, Ciccone W II, Radin A (1997) Percutaneous pinning of distal radius fractures: a biomechanical study. J Hand Surg [Am] 22(2):252-257

12. Short WH, Palmer AK, Werner FW, Murphy DJ (1987) A biomechanical study of distal radial fractures. J Hand Surg [Am] 12(4):529-534

13. Kreder HJ, Hanel DP, McKee M, Jupiter J, McGillivary G, Swiontkowski MF (1996) Consistency of AO fracture classification for the distal radius. J Bone Joint Surg Br 78(5):726-731

14. Andersen DJ, Blair WF, Steyers CM Jr, Adams BD, el-Khouri GY, Brandser EA (1996) Classification of distal radius fractures: an analysis of interobserver reliability and intraobserver reproducibility. J Hand Surg [Am] 21(4):574-582

15. Kurup HV, Mandalia V, Singh B, Shaju KA, Mehta RL, Beaumont AR (2005) Variables affecting stability of distal radial fractures fixed with $\mathrm{K}$ wires: a radiological study. Eur J Orthop Surg Traumatol 15(2):135-139 